Conclusion Compared with surgery alone, NCRT with cisplatin and 5-Fluorouracil does not improve overall survival but enhances postoperative mortality for patients with stage I or II OC (Clinical Trial.gov identifier NCT 00047112).

Funding Funded by the French ministry of Health-Programme Hospitalier de Recherche Clinique 1999.

Competing interests None declared

\section{OC-087 CLINICAL EVALUATION OF OESOPHAGEAL MUCOSAL INTEGRITY AND ACID SENSITIVITY IN PATIENTS WITH NERD. A STUDY USING BASAL IMPEDANCE AND ASSESSMENT OF MUCOSAL RECOVERY AFTER ACID CHALLENGE}

doi:10.1136/gutjnl-2012-302514a.87

P Woodland, ${ }^{*}$ M Al-Zinaty, I Bravi, J Jafari, D Sifrim. Barts and The London School of Medicine and Dentistry, Queen Mary University of London, London, UK

Introduction Patients with NERD have no erosions but impaired oesophageal mucosal integrity, that is, dilated intercellular spaces. It has been proposed that such microscopic changes can underlie symptoms in NERD, however, the relationship between impaired mucosal integrity and acid perception is unclear. Thus far, oesophageal mucosal integrity has been studied in vitro. Recently, oesophageal impedance basal values have been suggested as an in vivo surrogate to assess mucosal integrity in man. Low basal impedance is seen in patients with higher oesophageal acid exposure, and improves after PPI treatment. Oesophageal mucosal integrity, as expressed by basal impedance, is probably a dynamic process reflecting (1) the damaging effect of repeated acid reflux events and (2) the mucosal capacity to recover integrity. We hypothesised that there may be a relationship between mucosal integrity, recovery capacity and acid perception. We aimed to study the relationship between the dynamic properties of oesophageal mucosal integrity after acid challenge and symptom perception in patients with reflux symptoms.

Methods We studied 53 patients with typical reflux symptoms and no oesophagitis. A combined pH-MII catheter was inserted, and baseline distal oesophageal mucosal impedance measured for $15 \mathrm{~min}$ (and continuously thereafter). We performed a $10 \mathrm{~min}$ mid-oesophageal perfusion $(10 \mathrm{ml} / \mathrm{min})$ of a neutral solution. After a 10 min rest period an acid perfusion was performed with $\mathrm{pH} 1$ solution. Symptoms were recorded with a visual analogue scale. Impedance recovery was observed for $2 \mathrm{~h}$ post-acid perfusion in ambulatory conditions. Subjects then completed a $24 \mathrm{~h}$ reflux study.

Results There was significant inter-individual variability in preperfusion impedance baselines (mean 2059 , range 462-5388). Neutral perfusion caused a drop in impedance that recovered fully in $10 \mathrm{~min}$. Acid perfusion caused a drop in impedance that was slow to recover. The mean impedance recovery rate was $7.5 \Omega / \mathrm{min}$ (25th-75th percentile=3.1-10.9). 32 of 53 patients perceived heartburn during acid perfusion. Patients with slower impedance recovery $(<25$ th percentile, $\mathrm{n}=12)$ had lower basal impedance (mean \pm SEM $1331 \Omega \pm 256$ vs $3325 \Omega \pm 325, \mathrm{p}<0.01$ ), higher $24 \mathrm{~h}$ acid exposure $(5.2 \% \pm 1.0$ vs $1.7 \% \pm 0.3, \mathrm{p}<0.01)$, and more often acid sensitivity $(10 / 12$ vs $5 / 13, p<0.05)$ than those with faster impedance recovery ( $>75$ th percentile, $n=13$ ).

Conclusion A continuous impaired mucosal integrity (low impedance) might be a consequence of repeated reflux episodes with slow recovery. There is a link between mucosal integrity, recovery capacity and symptom perception. Low basal impedance and slow recovery after acid challenge are associated with increased acid sensitivity.

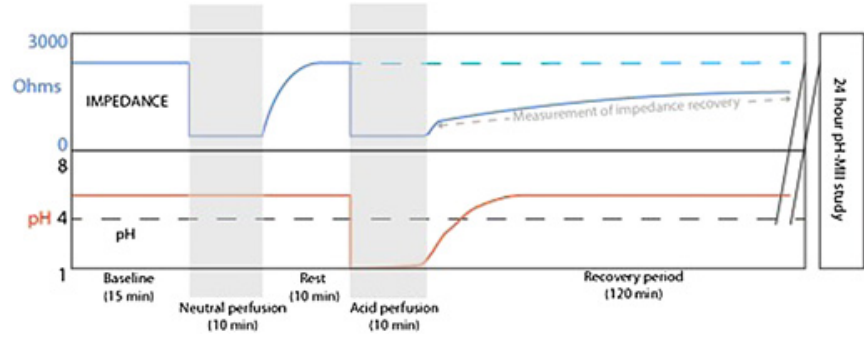

Abstract OC-087 Figure 1

Competing interests None declared.

\section{OC-088 IGE SENSITISATION TO FOOD AND INHALANT ALLERGENS IN UK ADULTS WITH EOE: A PILOT STUDY}

doi:10.1136/gutjnl-2012-302514a.88

1 J 0 Hayat, ${ }^{*}{ }^{2}$ J Skypala, ${ }^{3} \mathrm{~S}$ J Till, ${ }^{1} \mathrm{~J}$ Y Kang. 'Department of Gastroenterology, St.George's Hospital, London, UK; ${ }^{2}$ Department of Dietetics, Royal Brompton and Harefield NHS Foundation Trust, London, UK; ${ }^{3}$ Departments of Asthma, Allergy and Lung Biology, Kings College London, London, UK

Introduction Food and inhalant allergens have been implicated as triggers of eosinophilic oesophagitis (EoE). Although topical steroid therapy remains the mainstay of treatment in adults, elemental and six food elimination diets have been shown to decrease oesophageal eosinophilia and improve symptoms in children and more recently in adults. Limited data in North American adult EoE patients suggests that food allergens commonly associated with IgE sensitisation are peanut, egg and soy. We hypothesised that IgE sensitisation to foods and/or cross-reactive inhalant allergens plays a role in EoE. To test the hypothesis we designed a pilot study to explore possible relationships between IgE sensitisation to food/inhalant allergens and EoE in a UK adult population.

Methods Ten adult patients with biopsy-proven EoE ( $>15$ eosinophils/HPF) but no previously documented food allergy were included. Participants completed food allergy and dysphagia questionnaires, and underwent skin prick testing (SPT) to a battery of inhalant allergens: timothy grass, birch, six grasses, three trees, plane, mugwort, ragweed, Alternaria, Cladosporium, Aspergillus, house dust mite (HDM) and latex. Foods tested were milk, egg, prawn and cod and plant-derived foods: peanut, hazelnut, sesame, soy, mustard, corn, wheat, barley, celery, raw potato, apple, peach, grape, orange, tomato, melon, kiwi and strawberry. All SPT's were performed in the presence of positive (histamine) and negative (saline) controls. A wheal size $3 \mathrm{~mm}$ or more than the negative control was considered positive.

Results Of the 10 subjects ( $7 \mathrm{~m}$, median age 33 years, range $26-52$ ) who completed the study, eight reported dysphagia to solids nine times or more in the previous month. Two patients had required hospital admission in the previous month. Nine subjects identified one or more specific foods as a trigger for symptoms. The most commonly cited foods that were thought to trigger symptoms were meat (lamb or chicken) in four, nuts in three and citrus fruits or apples in three. Nine subjects had positive skin tests to both grass pollen and HDM; four of these subjects also had positive tests to at least three other inhalant allergens. These four subjects had the highest number of positive skin tests to foods (median of 9). The most common positive food SPTs were barley (7), wheat (5) and potato (4).

Conclusion The high prevalence of IgE sensitisation to foods in this pilot study supports our hypothesis that this plays a role in adult EoE pathophysiology. The high rates of barley and wheat sensitisation raise the possibility of IgE crossreactivity with homologous plant allergens in EoE, notably grass pollen. Further larger studies 
will be needed to confirm and investigate the significance of these findings.

Competing interests None declared.

\section{OC-090 DIFFERENT EFFECTS OF FODMAP (FERMENTABLE OLIGO-, DI-, AND MONO-SACCHARIDES, AND POLYOLS) COMPONENTS ON SMALL BOWEL WATER CONTENT: AN MRI STUDY}

doi:10.1136/gutjnl-2012-302514a.90 doi:10.1136/gutjnl-2012-302514a.89

${ }^{1} Y$ Y Lee, ${ }^{2}$ J R H Whiting, ${ }^{1} E$ V Robertson, ${ }^{1} \mathrm{M} H$ Derakhshan, ${ }^{1} \mathrm{~A}$ A Wirz, ${ }^{1} \mathrm{~A}$ Kelman, ${ }^{3} \mathrm{D}$ Morrison, ${ }^{2} \mathrm{P}$ Connolly, ${ }^{1} \mathrm{~K}$ E L McColl. ${ }^{1}$ Institute of Cardiovascular and Medical Sciences, University of Glasgow, Glasgow, UK; ${ }^{2}$ Department of Bioengineering, University of Strathclyde, Glasgow, UK; ${ }^{3}$ Stable Isotope Biochemistry Laboratory, Scottish Universities Environmental Research Centre, Glasgow, UK

Introduction In hiatus hernia there is proximal displacement of the gastro-oesophageal junction (GOJ) relative to the crural diaphragm impairing barrier function. Fluoroscopic studies have indicated proximal migration of the GOJ during TLOSRs but detailed study has been limited by radiation exposure. Using a non-radiological technique we have performed detailed examination of the GOJ during TLOSRs in healthy volunteers.

Methods In twelve subjects, a small magnet $(2 \times 1 \mathrm{~mm})$ was endoscopically clipped to the GOJ and the combined assembly of Hall Effect probe and 36 channel high resolution manometer (Sierra Scientific Inc., USA) was passed nasally. After a 600-calorie test meal (Fortisip, Nurticia, UK), the subjects were studied for $90 \mathrm{~min}$.

Results There was a median of 5 TLOSRs from each of the 12 volunteers. The median (range) amplitude and duration of GOJ migration from start to end of TLOSRs was $4.34 \mathrm{~cm}(1.6-8.8)$ and 23.63s (11.3-41.6) respectively. The proximal migration of GOJ during a TLOSR was characterised by a slow start (phase A) before accelerating (phase B) to peak amplitude. Descent of GOJ started off quickly (phase C) before slowing down (phase D) to baseline. These four different phases of movement are confirmed using mathematical modelling. The polynomial equation (1) and its differential (2) allow the calculation of maximum rate achievable for the four different phases using Microsoft Excel 2010 software:

$$
y=a x^{6}+b x^{5}+c x^{4}+d x^{3}+e x^{2}+f x
$$

$$
d y / d x=6 a x^{5}+5 b x^{4}+4 c x^{3}+3 d x^{2}+2 e x+f
$$

where $y$ is amplitude $(\mathrm{cm}), \mathrm{dy} / \mathrm{dx}$ is the differential of $\mathrm{y}, \mathrm{x}$ is time (s) and a to $f$ are non-linear regression constants. The median (range) velocity of phase $\mathrm{C}$ was $0.92 \mathrm{~cm} / \mathrm{s}(0.33-2.35)$ and this was faster when compared to phase B $0.67 \mathrm{~cm} / \mathrm{s}(0.23-1.73)$, phase D $0.36 \mathrm{~cm} / \mathrm{s}(0.06-0.99)$ and phase A $0.30 \mathrm{~cm} / \mathrm{s}$ $(0.03-2.31)$ respectively; $p<0.0001$. Phase $C$ velocity was strongly correlated with amplitude of GOJ migration with a correlation coefficient of $0.84(p=0.0001)$ but not the other three phases. None of the phases correlated with duration of GOJ migration during TLOSRs.

Conclusion The marked proximal movement of GOJ during TLOSRs represents transient and very severe herniation of the GOJ. Our study also suggests that the initial return of the GOJ to the diaphragmatic hiatus following TLOSRs may be controlled by elastic recoil of the phreno-oesophageal ligament.

Competing interests None declared
${ }^{1} \mathrm{~K}$ Murray, ${ }^{*} \mathrm{~V}$ Wilkinson-Smith, ${ }^{2} \mathrm{C}$ Lam, ${ }^{1} \mathrm{C}$ Hoad, ${ }^{1} \mathrm{E}$ Cox, ${ }^{1} \mathrm{C}$ Costigan, ${ }^{2} \mathrm{~L}$ Marciani, ${ }^{1} \mathrm{P}$ Gowland, ${ }^{2} \mathrm{R}$ Spiller. ${ }^{1}$ Sir Peter Mansfield Magnetic Resonance Centre, University of Nottingham, Nottingham, UK; ${ }^{2}$ NIHR Biomedical Research Unit, Nottingham Digestive Diseases Centre, Nottingham University Hospitals, Nottingham, UK

Introduction A randomised placebo controlled trial (RCT) has shown that FODMAPs exacerbate irritable bowel syndrome (IBS) symptoms in selected patients. FODMAPs are heterogeneous chemically, the major components being fructose and fructans. Both are poorly absorbed in the small bowel but fructose exerts a much greater osmotic effect than fructans which are fructose polymers. Whether this alters the overall impact is unknown. This study aims to test the hypotheses that (a) fructose will increase small bowel water content (SBWC) more than fructans; (b) adding glucose to fructose will reduce its effect, and (c) fructans will exert most of their effect as a result of colonic fermentation.

Methods 4-way, randomised, single blind, crossover study. 16 healthy volunteers underwent abdominal scans in a $1.5 \mathrm{~T} \mathrm{MRI}$ scanner after an overnight fast. They were then fed $500 \mathrm{ml}$ of water containing $40 \mathrm{~g}$ glucose, fructose, fructan or $40 \mathrm{~g}$ glucose plus $40 \mathrm{~g}$ fructose and underwent scanning and breath $\mathrm{H}_{2}$ measurement and completed bowel symptom questionnaires at hourly intervals for $5 \mathrm{~h}$. SBWC was measured from heavily T2 weighted magnetic resonance scans. 1-way analysis of variance was used.

Results (Mean \pm SEM) All drinks were well tolerated with only minor symptoms of gas, bloating, abdominal pain and diarrhoea reported. Relative to glucose, fructose increased SBWC significantly $(184 \pm 35 \mathrm{ml}$ vs $118 \pm 24 \mathrm{ml}$ glucose, $\mathrm{p}=0.001)$, while fructans had little effect ( $\mathrm{SBWC}=119 \pm 23 \mathrm{ml}, \mathrm{p}=0.7$ vs glucose). Adding $40 \mathrm{~g}$ glucose to $40 \mathrm{~g}$ fructose decreased SBWC $(145 \pm 27 \mathrm{ml}$, but the difference was not significant, $\mathrm{p}=0.08$ vs fructose). Breath $\mathrm{H}_{2}$ was initially higher for fructose than the other three solutions. (AUC was $8959 \pm 1880 \mathrm{ppm} . \mathrm{min}$ ) with fructans having the largest AUC at $14987 \pm 2568$ ppm.min). Values returned to baseline for fructose over the $5 \mathrm{~h}$ study, but increased to a maximum for fructan after $300 \mathrm{~min}$. Glucose had no effect on breath $\mathrm{H}_{2}$ concentration, but adding an equivalent amount to fructose significantly reduced breath $\mathrm{H}_{2}$ relative to fructose alone $(\mathrm{p}=0.0006)$.

Conclusion FODMAPs exert a variety of GI responses. Fructose but not fructans increases SBWC relative to glucose. Both fructose and fructans are malabsorbed as confirmed by breath $\mathrm{H}_{2}$ measurements. Fructose malabsorption is significantly reduced by addition of glucose. These different gastrointestinal responses can be readily identified using our non-invasive MRI techniques.

Competing interests None declared.

\section{OC-091 ONDANSETRON SLOWS TRANSIT AND IMPROVES STOOL CONSISTENCY IN PATIENTS WITH DIARRHOEA PREDOMINANT IRRITABLE BOWEL SYNDROME}

doi:10.1136/gutjnl-2012-302514a.91

${ }^{1} \mathrm{~K}$ Garsed, ${ }^{*} \mathrm{M}$ Hastings, 'L Marciani, ${ }^{1} \mathrm{C}$ Lam, ${ }^{1} \mathrm{G}$ Singh, ${ }^{1} \mathrm{M}$ Lingaya, ${ }^{2} \mathrm{P}$ Whorwell, ${ }^{1} \mathrm{R}$ Banwait, ${ }^{1} \mathrm{R}$ Spiller. ${ }^{1}$ Nottingham Digestive Diseases Centre NHIR BRU, University of Nottingham, Nottingham, UK; ${ }^{2}$ Neurogastroenterology and Motility, Wythenshawe Hospital, Manchester, UK

Introduction 5-Hydroxytryptamine three receptor antagonists (5HT3RA) are effective in diarrhoea predominant irritable bowel syndrome (IBS-D), with a number needed to treat (NNT) for 